MACROMOLECULES 46: 5337-5344 (2013).

DOI: $10.1021 / \mathrm{ma} 400535 \mathrm{r}$

\title{
Thermally Responsive Amphiphilic Conetworks and Gels Based on Poly(N-isopropylacrylamide) and Polyisobutylene
}

\author{
Gergely Kali ${ }^{1^{*}}$, Szilvia Vavra ${ }^{2}$, Krisztina László ${ }^{2}$, Béla Iván ${ }^{1}$ \\ ${ }^{1}$ Department of Polymer Chemistry, Institute of Materials and \\ Environmental Chemistry, Research Centre for Natural \\ Sciences, Hungarian Academy of Sciences, H-1525 Budapest, \\ P. O. Box 17, Hungary \\ ${ }^{2}$ Department of Physical Chemistry and Material Science, \\ Budapest University of Technology and Economics, $\mathrm{H}-1521$ \\ Budapest, Hungary
}

*Corresponding authors. E-mail:kali.gergely@ttk.mta.hu, ivan.bela@ttk.mta.hu 


\begin{abstract}
Novel amphiphilic conetworks (APCN) consisting of thermoresponsive poly $(\mathrm{N}$ isoproplyacrylamide) (PNiPAAm) cross-linked by hydrophobic methacrylate-telechelic polyisobutylene (MA-PIB-MA) were successfully synthesized in a broad composition range. The resulting PNiPAAm-I-PIB conetworks (" $"$ " stands for "linked by") were obtained by radical copolymerization of NiPAAm with MA-PIB-MA in tetrahydrofuran, a cosolvent for all the components. Low amounts of extractables substantiated efficient network formation. The composition dependent two glass transition temperatures $\left(T_{g}\right)$ by DSC analysis indicate microphase separation of the cross-linked components without mixed phases. It was found that the PNiPAAm-I-PIB conetworks are uniformly swellable in both water and $n$-hexane, i. e. these new materials behave either as hydrogels or as hydrophobic gels in aqueous or nonpolar media, respectively. The uniform swelling in both polar and nonpolar solutes indicates cocontinuous (bicontinuous) phase morphology. The equilibrium swelling degrees $(R)$ depend on composition, that is, the higher the PIB content, the lower the $\mathrm{R}$ in water and the higher in $\mathrm{n}$-hexane. The PNiPAAm phase keeps its thermoresponsive behavior in the conetworks as shown by significant decrease of the swelling degree in water between 20 and $35^{\circ} \mathrm{C}$. The lower critical solubility temperature (LCST) values determined by DSC are found to decrease from $34.1{ }^{\circ} \mathrm{C}$ (for the pure PNiPAAm homopolymer) to the range of $25-28{ }^{\circ} \mathrm{C}$ in the conetworks, and the extent of the LCST decrease is proportional with the PIB content. This indicates that PNiPAAm-I-PIB conetworks with predetermined and thermoresponsive swelling behavior can be designed and utilized in several advanced applications on the basis of results obtained in the course of this study.
\end{abstract}

Keywords: poly(N-isopropylacrylamide), polyisobutylene, amphiphilic conetwork and gel, bicontinuous morphology, LCST, thermoresponsive 


\section{INTRODUCTION}

Since its first description in the late $1950 \mathrm{~s}^{1}$, the thermoresponsive macromolecule, poly(N-isopropylacrylamide) (PNiPAAm) is one of the most widely investigated polymer described in the scientific literature. ${ }^{1-12}$ This polymer in aqueous solution, as well as its gel in swollen state, is sensitive to the temperature of the environment. ${ }^{2,3}$ Above a certain temperature, the hydrophilic PNiPAAm changes its philicity, rapidly becomes hydrophobic, ${ }^{4}$ and the polymer and the gel precipitates and collapses, respectively. ${ }^{2,3}$ This temperature, the so called lower critical solubility temperature (LCST), is around $32{ }^{\circ} \mathrm{C}^{1}$ for this polymer, which is close to the temperature of the human body. The combination of this thermoresponsive behavior with the biocompatibility of PNiPAAm makes it interesting for a large variety of applications, for example in the fields if biology, medicine or sensors. Therefore, not only basic, but also application oriented research have been carried out to investigate its properties and application possibilities, ranging from biomedicine to generally applicable objects. Examples for the biomedical applications include controlled drug delivery, ${ }^{5}$ biomaterials ${ }^{6}$, cell culture surfaces ${ }^{7}$, tissue engineering ${ }^{8}$ or biomolecule separation ${ }^{9,10}$, while some other uses, such as core/shell nanoparticles ${ }^{11}$ or intelligent glass ${ }^{12}$ are also interesting possibilities. However, one substantial disadvantage of the traditionally synthesized, pure PNiPAAm gels from practical point of view is related to the fact that these are mechanically not stable, soft, untreatable and nontransparent materials.

Amphiphilic conetworks (APCN) and their gels are mechanically more stable than their pure homopolymeric analogues. ${ }^{13,14}$ These novel, rapidly emerging materials, as displayed in Figure 1, consist not only of hydrophilic, but hydrophobic polymer 
components as well, which are covalently bonded together in a cross-linked macromolecular assembly. ${ }^{13-18}$ APCNs have some extraordinary properties, such as swelling with no dependence on solvent polarity ${ }^{13-17}$, nanophase separated structure ${ }^{13,15}$, biocompatibility $^{16}$ and extreme mechanical strength ${ }^{13,17}$, which make them applicable in many fields of life and material sciences and technologies ${ }^{18}$. It is noteworthy that there are only few publications on PNiPAAm- based APCNs. ${ }^{19}$ Lequieu and Du Prez prepared poly(N-isopropylacrylamide)-l-poly(tetrahydrofuran) $\quad$ (PNiPAAm-I-PTHF), ${ }^{19 a}$ while Gallardo et al. reported on poly(N-isopropylacrylamide)-I-poly(methyl methacrylate) (PNiPAAm-I-PMMA) ${ }^{19 b}$ conetworks. The LCSTs were determined in both cases gravimetrically or by differential scanning calorimetry (DSC). These measurements indicate that tuning the LCST of PNiPAAm in these conetworks can be achieved to a limited extent with selecting proper composition of such APCNs. In the case of PNiPAAm-I-PTHF conetworks ${ }^{19 a}$, the glass transition temperatures $\left(T_{g}\right)$ were also determined, and in some cases only one $T_{g}$ was found indicating miscibility of PTHF and PNiPAAm. It has to be mentioned that significant research activities are going on to modify the properties of PNiPAAm by other approaches as well, i. e. by constructing a variety of amphiphilic PNiPAAm containing polymer architectures, such as blocks, grafts, star-blocks, core/shell systems, interpenetrating networks etc. (see e. g. Refs. 20a-i and references therein).

Synthesis of PNiPAAm-based conetworks with other, for instance polyisobutylene (PIB) hydrophobic component was attempted only once with no obvious proof of success and with the lack of considerable characterization. ${ }^{21}$ Such kind of materials can be interesting not only from academic but even from application point of views as well. 
PIB is a fully saturated, rubbery, biocompatible and extremely hydrophobic polymer. ${ }^{22}$ Its unique properties, such as stability against chemical and physical impacts, flexibility, gas barrier characteristics and biocompatibility make it useful for a broad range of applications.

Herein, we report on the successful synthesis and systematic characterization of poly(N-isopropylacrylamide)-I-polyisobutylene (PNiPAAm-I-PIB; "I" stands for "linked by") conetworks by the so called macromonomer method, i. e. by copolymerizing $\mathrm{N}$ isopropylacrylamide with a well-defined methacrylate-telechelic PIB macromonomer prepared via quasiliving carbocationic polymerization.

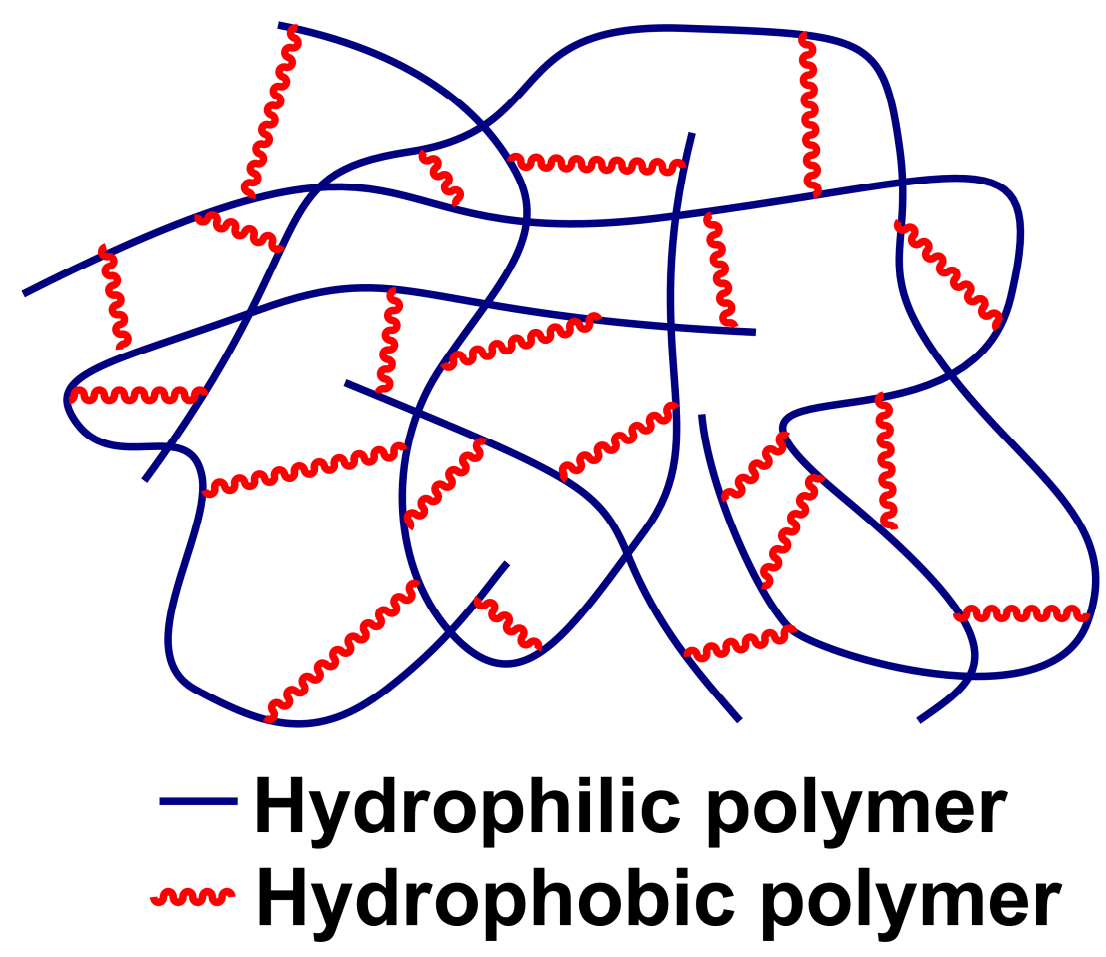

Figure 1. The schematic structure of amphiphilic polymer conetworks. 


\section{EXPERIMENTAL}

\section{Materials}

Methylmagnesium bromide (3.0 M solution in diethyl ether), 5-tert-butylisophthalic acid (98\%), ammonium chloride (puriss), titanium tetrachloride $\left(99.9 \%, \mathrm{TiCl}_{4}\right), 1,1,4,4-$ tetramethylethylenediamine (99.5\%, TMEDA), 9-borabicyclo[3.3.1]nonane (9-BBN; 0.5 M solution in THF), hydrogen peroxide (35\% solution in water), methacryloyl chloride $(97 \%, \mathrm{MACl})$, triethylamine $\left(99 \%, \mathrm{Et}_{3} \mathrm{~N}\right)$, calcium hydride $\left(90-95 \%, \mathrm{CaH}_{2}\right), \quad \mathrm{N}-$ isopropylacrylamid (97\%, NiPAAm) and aluminium oxide (activated, neutral) were all purchased from Aldrich. Hexane $(96 \%, \mathrm{Hx})$ was obtained from Scharlau S. A. Dichloromethane (99.8\%, DCM), tetrahydrofuran (99.8\%, THF) and methanol $(99.8 \%)$ were purchased from Chemolab. Isobutylene (IB, 99.8\%) and hydrogen chloride $(\mathrm{HCl}$, 99.8\%) were obtained from Messer Griesheim. Triethylamine ( $\mathrm{Et}_{3} \mathrm{~N}, 99 \%$ ) and potassium hydroxide (98\%) were purchased from Merck, while calcium chloride (anhydrous), allyltrimethylsilane (ATMS, 97\%) and a, $\alpha^{6}$-azobisisobutyronitrile (AIBN; $98+\%)$ were purchased from Fluka.

\section{Methods}

\section{Synthesis of methacrylate-telechelic polyisobutylene macromonomer}

The bifunctional carbocationic initiator tert-butyldicumyl chloride was synthesized according to the literature ${ }^{23}$ and was recrystallized twice before use. The methacrylatetelechelic PIB macromonomer (MA-PIB-MA) was synthesized by quasiliving carbocationic polymerization $^{22,24}$ of isobutylene. The initiator system was tertbutyldicumyl chloride/ $\mathrm{TiCl}_{4}$ and the nucleophilic additive was 1,1,4,4- 
tetramethylethylenediamine. The polymerization reaction was terminated by allyltrimethylsilane yielding quantitative end group functionalized allylic-telechelic PIB. ${ }^{22,23}$ After the allylation, three end-group modification steps were carried out. First, the allylic chain ends were modified by hydroboration, then oxidation of the chain ends was carried out by $\mathrm{H}_{2} \mathrm{O}_{2}$ in alkaline conditions $(\mathrm{KOH})$ in the second step leading to hydroxyl-telechelic PIB. ${ }^{23,24}$ Finally, a methacrylation step with methacryloyl chloride yielded the MA-PIB-MA macromonomer. The macromonomer was characterized in terms of terminal functionality and molecular weight distribution by ${ }^{1} \mathrm{H}$ NMR spectroscopy and gel permeation chromatography (GPC), respectively.

\section{Preparation of conetworks}

The bifunctional macromonomer (MA-PIB-MA) was copolymerized with the $\mathrm{N}$ isopropylacrylamide comonomer. The copolymerization was carried out via free radical copolymerization initiated by AIBN radical initiator in THF as common solvent of the components. The desired amounts of the bifunctional macromonomer, and the comonomer NiPAAm (in all cases $1 \mathrm{~g}$ of total mass), initiator and common solvent (THF) were measured into glass vials. The AIBN concentration was adjusted to the total concentration of the comonomers in order to keep the [comonomer]/[AIBN] ${ }^{0.5}$ ratio constant at the value of 0.99 value (see Table S1 in the Supporting Information for the applied amounts). The reaction mixtures were purged with nitrogen to remove oxygen and to homogenize the solutions. Then, the vials were placed and kept in an oven at 65 ${ }^{\circ} \mathrm{C}$ for 3 days. After the reaction, the vials were let to cool down to room temperature, the solvent was evaporated and the conetworks were dried under vacuum overnight. 
The resulting materials were placed in THF for two weeks to extract the unreacted materials (monomers, oligomers, polymers and initiator). The amounts of the extracted soluble parts were determined gravimetrically.

\section{Characterization methods}

\section{Gel Permeation Chromatography}

The average molecular weights of the macromonomer were measured by gel permeation chromatography (GPC). The GPC was equipped with RI, differential viscometer (Viscotec) and laser light scattering (Wyatt Co.) detectors. THF was used as mobile phase with a flow rate of $1.5 \mathrm{~mL} / \mathrm{min}$. Universal calibration was used to evaluate the GPC chromatograms.

\section{${ }^{1} \mathrm{H}$ NMR spectroscopy}

The endgroup functionality of the linear PIB macromonomer was investigated by ${ }^{1} \mathrm{H}$ NMR spectroscopy after the polymerization and every chain end modification. The ${ }^{1} \mathrm{H}$ NMR measurements were performed by a Varian 400 equipment $(400 \mathrm{MHz})$ in $\mathrm{CDCl}_{3}$ and at $30{ }^{\circ} \mathrm{C}$.

\section{Elemental Analyses}

The compositions of the conetworks were investigated by elemental analyses using Hereaus CHN-O-Rapid apparatus with $\mathrm{CuO}$ catalyst. 


\section{Differential Scanning Calorimetry}

A Mettler TG50 instrument was used for differential scanning calorimetry (DSC) analysis to determine the glass transition temperatures of the synthesized polymers. The midpoint of the specific heat increase in the transition region during the second heating is reported as the glass transition temperature $\left(T_{g}\right)$. Programmed heating cycles from $120^{\circ} \mathrm{C}$ to $200^{\circ} \mathrm{C}$ were used at a heating rate of $10^{\circ} \mathrm{C} / \mathrm{min}$ under nitrogen atmosphere.

The LCST values were determined on a MicroDSCIII apparatus (SETARAM). In these DSC measurements, the dry gel samples were contacted with Millipore water and kept at $\mathrm{T}=5^{\circ} \mathrm{C}$ for $2 \mathrm{~h}$ to obtain a stable baseline. Then the samples were heated up to $50^{\circ} \mathrm{C}$ with a scanning rate of $0.5^{\circ} \mathrm{C} / \mathrm{min}$.

\section{Swelling Measurements}

After the extraction, the conetwork samples were dried to constant weight in vacuum at room temperature, and then were cut into small pieces $\left(1-2 \mathrm{~cm}^{3}\right)$. The mass of the dry samples were weighted, and transferred into organic solvent or water. The mass of the samples were measured from time to time until the equilibrium was reached. The equilibrium swelling degree $(R)$ was calculated as the mass of solvent uptakes $\left(m_{\text {swollen- }}\right.$ $\left.m_{\text {dry }}\right)$ divided by the mass of the dry sample $\left(m_{\text {dry }}\right)\left(R=\left(m_{\text {swollen }}-m_{\text {dry }}\right) / m_{\text {dry }}\right)$. 


\section{RESULTS AND DISCUSSION}

\section{Synthesis of Telechelic MA-PIB-MA Macromonomer and PNiPAAm-I-PIB Conetworks}

The methacrylate-telechelic polyisobutylene (PIB) macromonomer (MA-PIB-MA) was synthesized by quasiliving carbocationic polymerization ${ }^{22}$ of isobutylene followed by three endgroup modification steps. First, allyl-telechelic PIB was obtained by direct endquenching of living difunctional PIB chain ends with allyltrimethylsilane. ${ }^{23}$ Then, hydroboration with 9-BBN followed by oxidation with $\mathrm{H}_{2} \mathrm{O}_{2}$ resulted in hydroxyl-telechelic PIB. ${ }^{23,24}$ Quantitative esterification of this macrodiol with methacryloyl chloride led to MA-PIB-MA with close to theoretical number average molecular weight and narrow molecular weight distribution $\left(M_{n}=4100, M_{w} / M_{n}=1.08\right)$, and chain end functionality of 2.0 according to GPC and ${ }^{1} \mathrm{H}$ NMR analyses, respectively (see Figure S1 for the GPC chromatogram in the Supporting Information).

The synthesis route of poly(N-isopropylacrylamide)-I-polyisobutylene (PNiPAAmI-PIB; "I" abbreviates "linked by") is shown in Scheme 1. For the successful synthesis of the planned conetwork series, a cosolvent has to be used due to the immiscibility of the hydrophilic (polar) NiPAAm monomer and PNiPAAm and the hydrophobic (nonpolar) PIB. An appropriate cosolvent can dilute all the components to prevent phase separation during the network forming reaction. In our case, tetrahydrofuran (THF) is proved to be a suitable solvent which can accomplish this requirement. Some other basic requirements have to be also met for successful conetwork synthesis. First, the two components, i. e. the low molecular weight monomer and the telechelic macromonomer, should be copolymerizable. Second, the polymer chains formed from the low molecular weight 
comonomer have to be long enough to incorporate at least two macromonomer crosslinkers. The first requirement is fulfilled because methacrylates and acrylamides are copolymerizable, while the second can be accomplished by the optimal choice of the monomer/initiator ratios considering the $\mathrm{DP}_{\mathrm{n}} \sim[\mathrm{M}] /[\mathrm{I}]^{0.5}$ relationship in radical polymerizations (where $\mathrm{DP}_{\mathrm{n}},[\mathrm{M}]$ and $[\mathrm{I}]$ stand for the degree of polymerization, monomer and initiator concentrations, respectively). As to the copolymerization of NiPAAm with the methacrylate telechelic MA-PIB-MA macromolecular cross-linker, it has to be noted that the copolymerization reactivity ratios $(r)$ are favorable for the incorporation of MAPIB-MA in the conetworks on the basis of literature values for copolymerization between NiPAAm and methacrylates. According to literature data, the $r_{1}$ (NiPAAm) and $r_{2}$ values are 0.39 and 2.69 for NiPAAm and glycidyl methacrylate ${ }^{25}$ and 0.27 and 2.82 for NiPAAm and methyl methacrylate. ${ }^{26}$ This means that in spite of the decrease of the apparent copolymerization reactivity of the macromonomer in comparison with its low molecular weight counterparts, sufficient incorporation of the MA-PIB-MA macrocrosslinker is expected in the conetworks on the basis of recent findings in the course of similar conetwork syntheses. ${ }^{13 \mathrm{~h}}$ It is also noteworthy that the product of the copolymerization ratios of NiPAAm and methacrylates are close to one, which indicates random copolymerization, that is random incorporation of MA-PIB-MA cross-linker in the PNiPAAm chains. Taking these requirements consideration into account, a series of PNiPAAm-I-PIB conetworks was attempted with MA-PIB-MA contents between 20-70 wt\% as shown in Table 1 by copolymerizing NiPAAm with MA-PIB-MA by AIBN initiator in THF at $65^{\circ} \mathrm{C}$ for 72 hours. 
Right after the reaction, the resulting gels were placed in THF, a common solvent for the both polymers for a week in order to remove the unreacted soluble components. The extraction procedure was repeated once more, the extractables were combined, and the solvent was evaporated. The dried soluble fractions were lower than 10 wt $\%$ of the feed amount with the exception of the two lowest PIB containing samples with up to 30 wt\%. These results confirm efficient conetwork formation. The extractables were characterized by GPC and ${ }^{1} \mathrm{H}$ NMR spectroscopy, and the results showed that the soluble fraction for all the conetworks contained mainly the unreacted hydrophilic Nisopropylacrylamide monomer and its oligomer. The number average molecular weights of the extracted materials were lower than 400 with broad molecular weight distribution in all the cases.
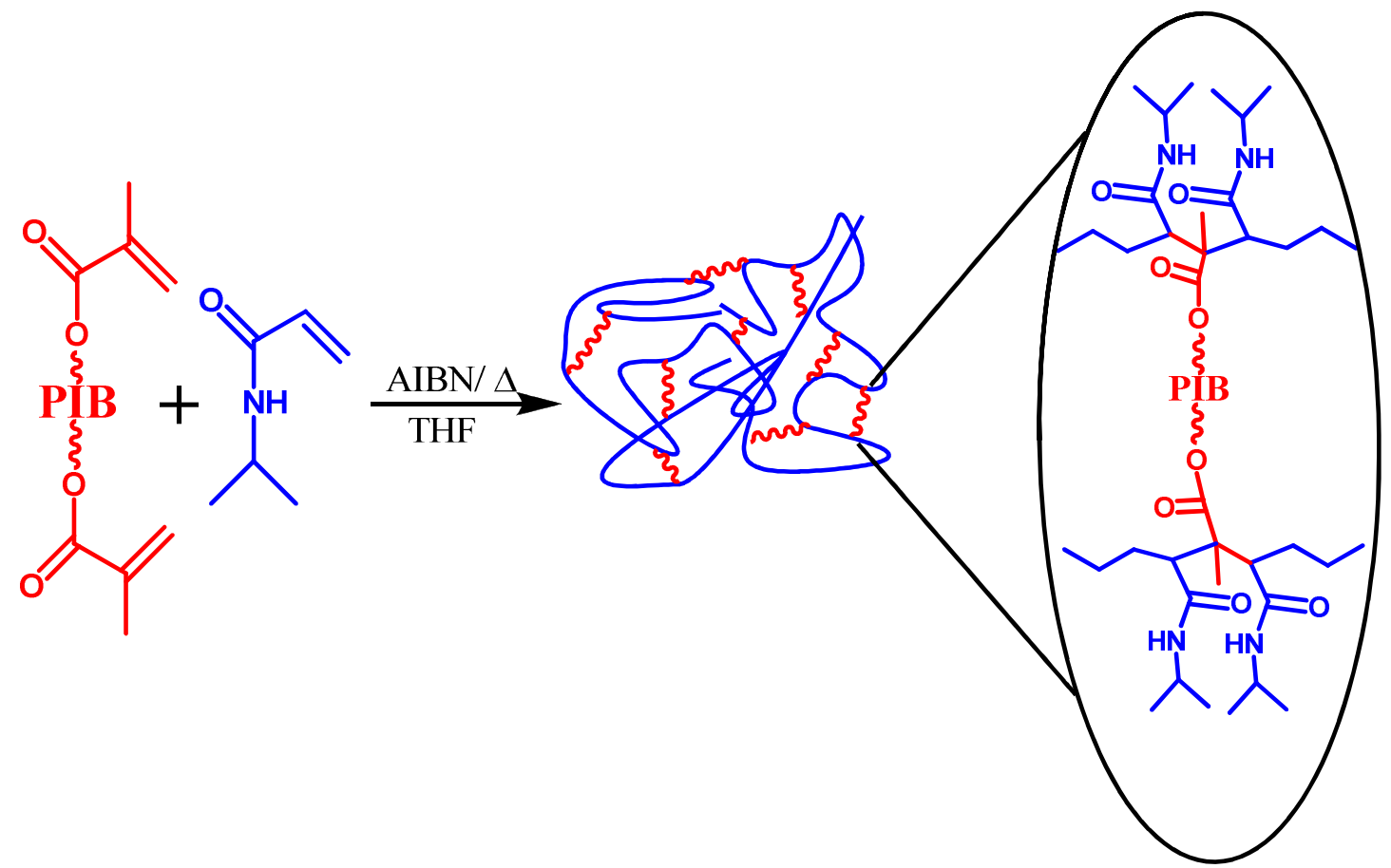

Scheme 1. The synthesis route of poly(N-isopropylacrylamide)-I-polyisobutylene (PNiPAAm-I-PIB) amphiphilic conetworks ("I" stands for "linked by"). 


\section{Characterization of the conetworks by elemental analysis and DSC measurements}

After extraction, all the conetworks were dried to constant weight in vacuo. The compositions of the resulting conetworks were determined by elemental analysis. The results of the elemental analysis, shown in Table 1, indicate successful synthesis of a series of PNiPAAm-I-PIB conetworks in a wide composition range, and conetworks with PIB content between 34 and 73 wt\% were obtained. The sample identification of these amphiphilic conetwork (APCN) samples is provided by their PIB content that is APCN43 means a PNiPAAm-I-PIB conetwork with 43 wt\% PIB content. As presented in Table 1, the PIB contents were slightly higher than the theoretical values (feed composition). This is in line with the analysis results of the extractables, i. e. with its high NiPAAm and oligo PNiPAAm content, on the one hand. On the other hand, it is also in agreement with the results of a recent study ${ }^{13 \mathrm{~h}}$ according to which local partition of the polar low molecular weight monomer between the solution and the vicinity of the terminal nonpolar macromonomer in the propagating polymer chain may increase the apparent reactivity of the macromonomer. As a result, the MA-PIB-MA macro cross-linkers efficiently incorporated in the PNiPAAm-I-PIB conetworks providing sufficient cross-linking density and low amounts of extractables. 
Table 1. The compositions of the copolymerization feed and the PNiPAAm-I-PIB conetworks (determined by elemental analysis) and the glass transition temperatures obtained by DSC.

\begin{tabular}{|l|c|c|c|c|c|c|}
\hline \multirow{2}{*}{ Sample } & \multicolumn{2}{|c|}{$\begin{array}{c}\text { Feed composition } \\
\text { (wt\%) }\end{array}$} & \multicolumn{2}{c|}{$\begin{array}{c}\text { Conetwork } \\
\text { composition } \\
\text { (wt\%) }\end{array}$} & \multicolumn{2}{c|}{$\mathbf{T}_{\mathrm{g}}{ }^{\circ}{ }^{\circ}$ C) } \\
\cline { 2 - 8 } & NiPAAm & PIB & NiPAAm & PIB & PNiPAAm & PIB \\
\hline APCN34 & 80 & 20 & 66 & 34 & 121 & -66 \\
\hline APCN36 & 70 & 30 & 64 & 36 & 128 & -61 \\
\hline APCN43 & 60 & 40 & 57 & 43 & 122 & -58.5 \\
\hline APCN59 & 50 & 50 & 41 & 59 & 122 & -58 \\
\hline APCN65 & 40 & 60 & 35 & 65 & 113 & -56 \\
\hline APCN73 & 30 & 70 & 27 & 73 & 107 & -57.5 \\
\hline PNiPAAm & - & - & 100 & - & 134 & - \\
\hline & - & - & - & 100 & - & -78 \\
\hline
\end{tabular}

The glass transition temperatures $\left(T_{g}\right)$, which are indicative on the miscibility of the PNiPAAm and PIB components in the conetworks, were determined by DSC. A representative DSC scan of these conetworks is displayed in Figure 2 (sample APCN59). As shown in this Figure 2 and in Table 1, the PNiPAAm-I-PIB conetworks 
exhibit two glass transitions. One appears in the region of the $\mathrm{T}_{\mathrm{g}}$ of $\mathrm{PIB}$ at low temperatures, while the other glass transition for the PNiPAAm component is observed at higher values, somewhat below that of the pure homopolymer. These findings indicate that both PNiPAAm and PIB form separate domains in the PNiPAAm-I-PIB conetworks, that is chemically connecting these two immiscible polymers with covalent bonds in a conetwork does not lead to mixing these two macromolecules. This means that the two components with different philicity in these conetworks form separate phases. Because the covalent bonds between the immiscible polymer chains in these cross-linked macromolecular assemblies prevent macroscopic phase separation, microphase separation occurs in these new materials. This is also supported by the fact that the PNiPAAm-I-PIB conetworks, and their gels as well, are transparent or faintly opaque, that is the average domain sizes are lower than that of the wavelength of the visible light. Similar findings have been reported for other conetworks composed of covalently bonded immiscible macromolecules. ${ }^{14,15,17}$

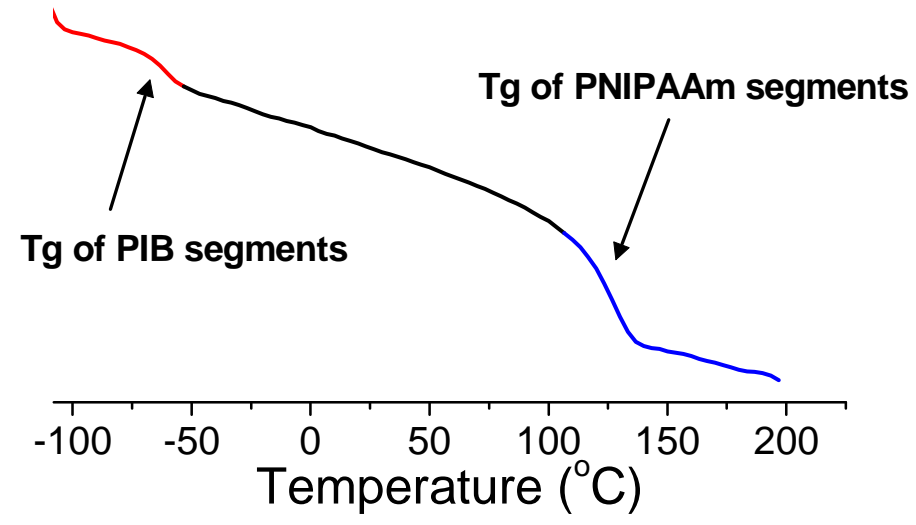

Figure 2. Differential scanning calorimetric thermogram of a PNiPAAm-I-PIB conetwork with 59 wt\% PIB content (second heating scan, $10{ }^{\circ} \mathrm{C} / \mathrm{min}$ heating rate). 
The dependence of the $T_{g} s$ on the composition is presented in Table 1 and Figure 3. As these data indicate, the $T_{g}$ of PIB in these bicomponent materials is higher than that of the starting homopolymer $\left(-78{ }^{\circ} \mathrm{C}\right)$. It can also be seen that the $\mathrm{T}_{g}$ of PIB in the conetworks varies between -66 and $-56{ }^{\circ} \mathrm{C}$, and increases with increasing PIB content up to about $40-50$ wt\% PIB content, then becomes nearly constant. In contrast, the $T_{g}$ of the PNiPAAm domains significantly decreases with increasing PIB content, i. e. with decreasing PNiPAAm content. These $\mathrm{T}_{\mathrm{g}}$ values are between 107 and $128{ }^{\circ} \mathrm{C}$, and considerably lower than that of the PNiPAAm homopolymer $\left(134{ }^{\circ} \mathrm{C}^{32}\right)$. In miscible polymer blends ${ }^{27,28}$, only one $\mathrm{Tg}$, depending on composition, between the $T_{g}$ of the components exists (see e. g. Refs. 27,28 and references therein). The observation of two $T_{g} s$ equal to that of the homopolymer components indicates immiscible systems. In the case of partial miscibility, the $T_{g} s$ of the blend composing polymers changes towards the $T_{g}$ of the other component by lowering their contents in the bicomponent polymer system. ${ }^{28}$ As shown in Figure 3 and in Table 1, while the $T_{g}$ of PNiPAAm decreases with its decreasing content in the PNiPAAm-I-PIB conetworks, the $T_{g}$ of PIB decreases and not increases with PIB content as it would be expected in the case of partial miscibility. Thus, partial miscibility of PNiPAAm and PIB in their conetworks can be excluded. As recent solid state NMR investigations ${ }^{131}$ with similar conetworks indicate that the covalent linkage and structural parameters of the conetworks, such as the extent of cross-linking, should play key role in determining the chain mobility in these novel polymeric materials. 


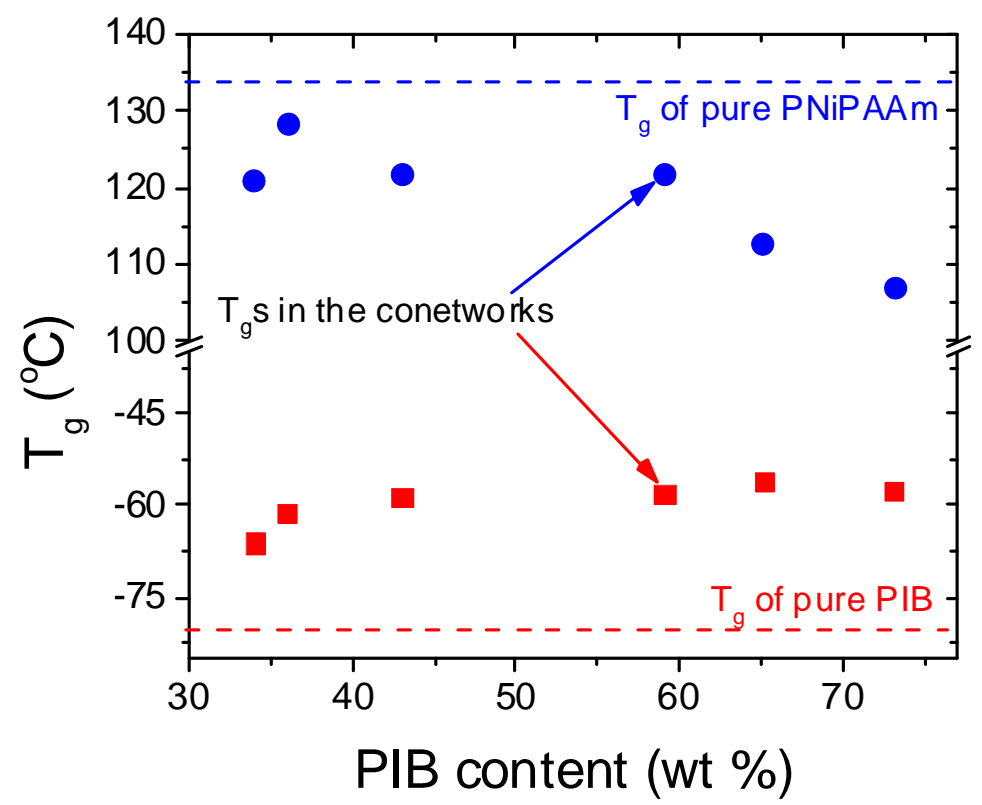

Figure 3. The glass transition temperature $\left(T_{g}\right)$ values as the function of the PIB content in the PNiPAAm-I-PIB conetworks ( $\square \mathrm{Tg}$ of PIB, $\bullet$ Tg of PNiPAAm in the conetworks).

\section{Swelling behavior of PNiPAAm-I-PIB conetworks}

One of the most interesting properties of amphiphilic conetworks is their swelling ability in polar and nonpolar solutes. Pieces of the extracted and dried PNiPAAm-I-PIB conetworks were transferred into water and $n$-hexane, and were let to swell until they reached the equilibrium. The mass of the swollen conetworks were measured, and the equilibrium swelling degrees $(R)$ were calculated. Figure 4 displays the resulting $R$ values as a function of conetwork composition. As shown in this Figure, the PNiPAAm-IPIB conetworks are able to swell in both polar (water) and nonpolar (hexane) solutes, that is, these new materials possess amphiphilic character. This means that depending on their outer environment, these conetworks behave either as hydrogels (in the presence of water) or hydrophobic gels (in case of hydrophobic, nonpolar solutes). 
Thus, both kinds of gels can be obtained from these new cross-linked polymer assemblies by selecting proper solvents. The data in Figure 4 also reveal that the swelling capacity of the PNiPAAm-I-PIB conetworks is composition dependent, and it also depends on the temperature when water is the swelling agent. As the hydrophobic PIB content increases, the equilibrium swelling degree decreases in water, and in contrast, the degree of swelling increases in $n$-hexane. It is interesting to observe that these conetworks are able to swell uniformly in both hexane and water in a broad composition range of $\sim 40-65$ wt\% PIB content. This uniform swelling capacity in solutes with opposite polar-nonpolar character indicates that both separate domains of PNiPAAm and PIB in the conetworks form cocontinuous (bicontinuous) phase structure, which provides the possibility of swelling in either aqueous or nonpolar solvents uniformly. Another important aspect of these novel PNiPAAm-I-PIB conetwork hydrogels is their observed mechanical and dimensional stability compared to the homopolymer PNiPAAm hydrogel with the same cross-linking density (for illustration, see for instance the picture of these gels in Figure S1 in the Supporting Information). 


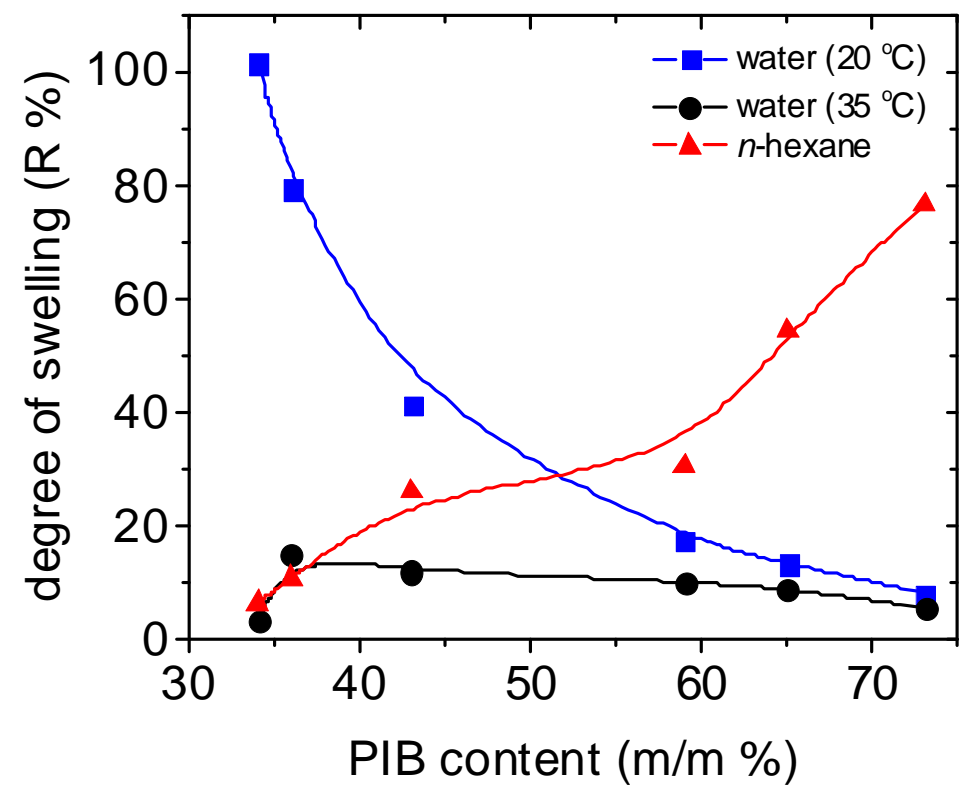

Figure 4. Equilibrium swelling degrees $(\mathrm{R})$ of the PNiPAAm-I-PIB conetworks as a function of PIB content in $n$-hexane and in water at $20^{\circ} \mathrm{C}$ and $35^{\circ} \mathrm{C}$.

The PNiPAAm homopolymer is a well-known thermoresponsive polymer with lower critical solution temperature (LCST) of $\sim 32-34{ }^{\circ} \mathrm{C}$ which has already been utilized in a variety of advanced applications (see e. g. Refs. 4-12,20 and references therein). Therefore, it is of paramount importance to investigate whether PNiPAAm keeps its thermoresponsive behavior in conjunction with the strongly hydrophobic PIB macrocross-linkers in the novel PNiPAAm-I-PIB conetwork hydrogels. In order to reveal this property, the conetworks were first swollen in water at $20^{\circ} \mathrm{C}$, and after reaching the swelling equilibrium, the temperature was raised to $35{ }^{\circ} \mathrm{C}$. As shown in Figure 4, the equilibrium swelling ratios decrease in all cases to low values (in the range of $10 \%$ or lower), and the amplitude of the change in the swelling degrees is composition dependent. Higher the PNiPAAm content, higher the extent of deswelling. For the 
conetworks with low PIB content, the equilibrium swelling degrees decreases by one order of magnitude, i. e. from $\sim 100 \%$ under $\sim 10 \%$ as the temperature cross the LCST. It is important to note that the observation of LCST of PNiPAAm in the PNiPAAm-I-PIB conetworks also clearly indicates the existence of independent non-mixed domains of PNiPAAm and PIB in these novel cross-linked polymeric materials. Due to the $\mathrm{H}$-bond formation between NiPAAm units and water in the conetworks below the LCST, the cross-linked PNiPAAm is in swollen state, and it behaves as hydrophilic polymer. Above this temperature, the hydrophobic interactions become thermodynamically more favorable than the polar ones, and the polymer becomes hydrophobic, i.e. PNiPAAm segments are not able to swell in aqueous solutions anymore. As a consequence, these conetworks are not only novel amphiphilic but smart thermoresponsive polymeric materials as well. At high PIB content, the deswelling of the gels with the increasing temperature is less significant. As published in the literature ${ }^{13,15}$ amphiphilic conetworks possess cocontinuous morphology only in a certain composition range. At high hydrophobic (or hydrophilic) content, the hydrophilic (or hydrophobic) components of the conetworks are present as dispersed phases. This can be one of the reasons of the infinitesimal deswelling of the conetwors with high PIB content. The other explanation is also the high hydrophobic content, which shifts dramatically the LCST of the conetworks to the low temperature region (even below $20^{\circ} \mathrm{C}$ ) and broadens the range of deswelling causes this negligible response. ${ }^{30}$

It is seen that all the above described gels response to the temperature change of the surrounding aqueous media. The swelling behavior of the conetworks is expected to show hysteresis during continuous heating and cooling cycles over the range of LCST. ${ }^{29}$ 
This phenomenon was investigated by measuring the changes of the equilibrium swelling degrees of the conetworks with increasing and decreasing temperatures. As the results show in Figure 5 for the selected APCN with $36 \%$ PIB content, the conetwork presents different LCSTs depend on heating, or cooling during the measurement. For this conetwork sample, while the temperature increases the PNiPAAm segments start to shrink at and around $25{ }^{\circ} \mathrm{C}$, and elongated deswelling occurs, ended around $40{ }^{\circ} \mathrm{C}$. From this point, the temperature was reversed and cooled down to $20{ }^{\circ} \mathrm{C}$. During this cooling the measured LCST was shifted to lower temperatures. This measured hysteresis is well known effect of PNiPAAm based homopolymer gels.

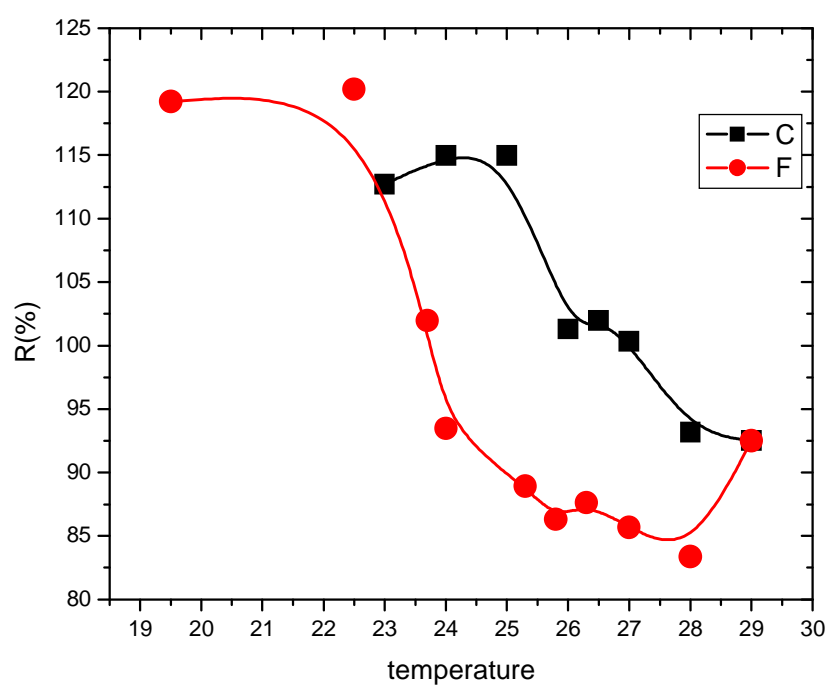

Figure 5. A representative temperature responsive swelling curve of PNiPAAm-/PIB conetwork, with $36 \%$ PIB content.

In order to determine the LCST of the PNiPAAm-I-PIB conetwork gels and a poly (N-isopropylacrylamide) homopolymer network cross-linked with N,N'-methylene-bis 
acrylamide, these were let to swell in deionized water, and their LCSTs were measured by differential scanning calorimetry. Figure 5 shows the resulting DSC thermograms, the minima of which belong to the LCST values. ${ }^{30}$ As shown in this Figure, the LCST values of PNiPAAm in the conetworks are between 25 and $28{ }^{\circ} \mathrm{C}$, and these are significantly lower than that of the pure PNiPAAm hydrogel $\left(34.1^{\circ} \mathrm{C}\right)$ measured by using the same conditions. The decrease of LCST of PNiPAAm by attached hydrophobic comonomers or groups is a well-known effect. ${ }^{31}$ In such instances, the overall hydrophobicity of the gel increases with increasing hydrophobic content, and the hydrophobic interactions become more favorable in water even at lower temperatures than $\mathrm{H}$-bond formation. As a consequence, lower LCSTs are observed. This trend is also clearly can be seen in the case of the PNiPAAm-I-PIB conetworks as well. As displayed in Figure 6, the LCSTs of the PNiPAAm phases are $27.6,26.7$ and $25.1{ }^{\circ} \mathrm{C}$ with 34,36 and 43 wt\% hydrophobic PIB cross-linkers in the conetworks. PIB reduces the LCST of PNiPAAm proportionally with the PIB content. The increasing hydrophobicity is also reflected in the slow DSC response curves. The pure PNiPAAm gel swollen in water exhibits an asymmetric DSC peak, and when the increasing temperature reaches the LCST an abrupt heat uptake is followed by a slow relaxation tail. ${ }^{32}$ In the PNiPAAm-I-PIB conetworks, the shift of the onset temperature is accompanied with a much more gradual progress of the endothermic peak. The shape of the peaks and their increasing half width indicate that the phase transition slows down, compared to the homopolymer gel, because the reorganization of the polymer chains/segments is hindered in the conetworks. A reduction in the thermal conductivity of the more hydrophobic system may also contribute to this effect. 


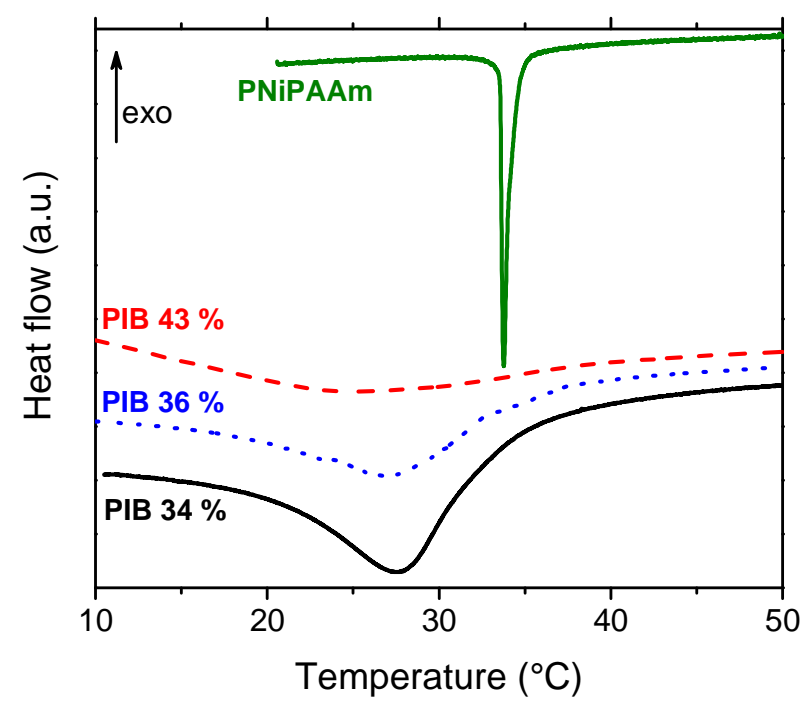

Figure 6. DSC response of a homopolymer PNiPAAm and PNiPAAm-I-PIB hydrogel samples in water (heating rate $0.05{ }^{\circ} \mathrm{C} / \mathrm{min}$; successive curves are shifted vertically for clarity.)

\section{Conclusions}

Successful synthesis of poly(N-isopropylacrylamide)---polyisobutylene (PNiPAAm-I-PIB) conetworks was carried out by free radical copolymerization of NiPAAm with methacrylate telechelic PIB macromonomer, and a series conetworks with a broad composition range was obtained. DSC measurements indicate the presence of two distinct $T_{g} \mathrm{~S}$, i. e. microphase separated structure of these new conetworks with PIB and PNiPAAm domains. Uniform swelling in both hydrophilic (water) and hydrophobic ( $\mathrm{n}$ hexane) solvents confirm cocontinuous (bicontinuous) domain structure and also the amphiphilic character of these novel bicomponent cross-linked macromolecular 
assemblies. This means that the PNiPAAm-I-PIB conetworks behave as hydrogels in aqueous and as hydrophobic gels in nonpolar environment. The PNiPAAm component does not loose its temperature responsive property, i. e. the existence of LCST, in the conetworks, which was proven by swelling and DSC measurements. The swelling in polar and nonpolar solvents, namely in water and in $n$-hexane, as well as the thermoresponsive behavior are found to be composition dependent. The LCST of these conetworks decreases to the range of $25-28{ }^{\circ} \mathrm{C}$ in comparison with $34.1{ }^{\circ} \mathrm{C}$ for the homopolymer network of PNiPAAm, and the rate of the phase transition also decreases in the hydrophobic sorroundings in the conetworks. These novel, dimensionally and mechanically stable thermoresponsive conetworks may find a variety of specific applications due their unique structure and properties.

\section{Acknowledgements.}

The authors are grateful for the elemental, thermal and GPC analyses to Dr. Hedvig Medzihradszky-Schweiger, Ms. Judit Szauer, Dr. Márta Szesztay and Ms. Erzsébet Tyroler, respectively. Financial support of this research by the Hungarian Scientific Research Fund (OTKA K75182 and K81592) is acknowledged. 


\section{References}

${ }^{1}$ W. C. Wooten, R. B. Blanton, H. W. Coover Jr, J. Polym. Sci., 1957, 25, 403.

${ }^{2}$ M. Heskins, J. E. Guillet, J. Macromol. Sci. Chem. 1968, 2, 1441.

${ }^{3}$ T. Tanaka, Phys. Rev. Lett., 1978, 40, 820.

${ }^{4}$ [a] K. László , A. Fluerasu , A. Moussaïd, E. Geissler Soft Matter, 2010, 6, 4335, [b]

K. László , A. Guillermo, A. Fluerasu , A. Moussaïd, E. Geissler Langmuir, 2010, 26,

4415, [c] C. H. Alarco'n, S. Pennadam, C. Alexander, Chem. Soc. Rev., 2005, 34, 276.

${ }^{5}$ [a] M. Yang, Y. Ding, L. Zhang, X. Qian, X. Jiang, B. Liu J. Biomed. Mater. Res., 2007, 81, 847. [b] F. Kohori, K. Sakai, T. Aoyagi, M. Yokoyama, M. Yamato, Y. Sakurai, T. Okano Coll. Surf, B Biointerfaces, 1999, 16, 195.

${ }^{6}$ [a] A. A. Naddaf, I. Tsibranska, H. J. Bart Chem. Eng. Proc., 2010, 49, 581, [b] S. Liu, Y. Yang, X. Liu, Y. Tong Biomacromolecules, 2003, 4, 1784, [c] H. Cheng, J. Zhu, Y. Sun, S. Cheng, X. Zhang, R. Zhuo Bioconjugate Chem., 2008, 19, 1368.

${ }^{7}$ [a] A. K. Brun-Graeppi, C. Richard, M. Bessodes, D. Scherman, O. W. Merten Prog. Polym. Sci., 2010, 35, 1311, [b] E. L. Lee, H.A. von Recum J Biomed. Mater. Res. A, 2010, 93, 411.

${ }^{8}$ G. Y. Huang, L. H. Zhou, Q. C. Zhang, Y. M. Chen, W. Sun, F. Xu, T. J. Lu Biofabrication, 2011, 3, 012001.

${ }^{9}$ K. Nagase, J. Kobayashi, T. Okano J. Royal Soc. Interface, 2009, 6, 293.

${ }^{10}$ H. Kanazawa, T. Okano J. Chrom. A, 2011, 1218, 8738.

11 C. Mangeney, F. Ferrage, I. Aujard, V. Marchi-Artzner, L. Jullien, O. Ouari, E. D. Rekai, A. Laschewsky, I. Vikholm, J. W. Sadowski J. Am. Chem. Soc., 2002, 124, 5811. 
${ }^{12}$ M. Zrinyi, A. Szilagyi, G. Filipcsei, J. Feher, J. Szalma, G. Moczar Polym. Adv. Technol., 2001, 12, 501.

${ }^{13}$ [a] G. Kali, T. K. Georgiou, B. Iván, C. S. Patrickios, E. Loizou, Y. Thomann, J. C. Tiller Macromolecules, 2007, 40, 2192. [b] G. Kali, T. K. Georgiou, B. Iván, C. S. Patrickios, E. Loizou, Y. Thomann, J. C. Tiller Langmuir, 2007, 23, 10746. (g) Fodor, Cs.; Kali, G.; Iván, B. Macromolecules 2011, 44, 4496-4502. (h) Fodor, Cs.; Iván, B. J. Polym. Sci. Part A: Polym. Chem. 2011, 49, 4729-4734. (i) Domján, A.; Fodor, Cs.; Kovács, Sz.; Marek, T.; Iván, B.; Süvegh, K. Macromolecules 2012, 45, 7557-7565.

14 [a] C.S. Patrickios, T. K. Georgiou Curr. Opi Colloid Interface Sci, 2003, 8, 76. [b] G. Erdodi, J. P. Kennedy Prog. Polym Sci. 2006, 31, 1. [c] L. Mespouille, J. L. Hedrick, P. Dubois Soft Matter. 2009, 5, 4892. [d] Kamata, H.; Chung, U.; Shibayama, M.; Sakai, T. Soft Matter 2012, 8, 2876. [e] Cui, J.; Lackey, M. A.; Madkour, A. E.; Saffer, E. M.; Griffin, D. M.; Bhatia, S. R.; Crosby, A. J.; Tew, G. N. Biomacromolecules 2012, 13, 584. [f] Rikkou-Kalourkoti, M.; Loizou, E.; Porcar, L.; Matyjaszewski, K.; Patrickios, C. S. Polym. Chem. 2012, 3, 105. [g] Domján, A.; Mezey, P.; Varga, J. Macromolecules 2012, 45, 1037. [h] Sugimoto, H.; Nishino, G.; Koyama, H.; Daimatsu, K.; Inomata, K.; Nakanishi, J. Appl. Polym. Sci. 2012, 124, 1316. [i] Sugimoto, H.; Nishino, G.; Tsuzuki, N.; Daimatsu, K.; Inomata, K.; Nakanishi, E. Colloid. Polym. Sci. 2012, 290, 173.

${ }^{15}$ [a] J. Scherble, R. Thomann, B. Iván, R. Mülhaupt J. Polym. Sci., Part B: Polym. Phys. 2001, 39, 1429. [b] B. Iván, K. Almdal, K. Mortensen, I. Johannsen, J. Kops Macromolecules 2001, 34, 1579. [c] A. Domján, G. Erdődi, M. Wilhelm, M. Neidhöfer, K. Landfester, B. Iván, H. W. Speiss Macromolecules 2003, 36, 9107. [d] B. Iván, M. Haraszti, G. Erdődi, J. Scherble, R. Thomann, R. Mülhaupt Macromol. Symp. 2005, 227, 
265. [e] N. Bruns, J. Scherble, L. Hartmann, R. Thomann, B. Iván, R. Mülhaupt, J. C. Tiller Macromolecules 2005, 38, 2431. [f] J. Tobis, Y. Thomann, J. C. Tiller Polymer 2010, 51, 35 .

${ }^{16}$ [a] J. P. Kennedy Macromol. Symp. 1994, 85, 76. [b] I. S. Isayeva, A. N. Gent, J. P. Kennedy J. Polym. Sci., Part A: Polym. Chem. 2002, 40, 2075. [c] R. Haigh, N. Fullwood, S. Rimmer Biomaterials 2002, 23, 3509. [d] S. Rimmer, M. J. German, J. Maughan, Y. Sun, N. Fullwood, J. Ebdon, S. MacNeil Biomaterials 2005, 26, 2219. [e] J.P. Kennedy, K.S. Rosenthal, B. Kashibhatla Des Monomers Polym 2004, 6, 485.

17 [a] G. Kali, T. K. Georgiou, B. Iván, C. S. Patrickios J. Polym. Sci., Part A: Polym. Chem., 2009, 47, 4289. [b] M. Haraszti, E. Tóth, B. Iván Chem. Mater. 2006, 18, 4952. 18 [a] B. Iván, J.P. Kennedy, P.W. Mackey ACS Symp. Ser. 1991, 469, 194., [b] B. Iván, J.P. Kennedy, P.W. Mackey ACS Symp. Ser. 1991, 469, 203., [c] B. Iván, J.P. Kennedy, P.W. Mackey US Patent 5,073,381, Dec 17, 1991. [d] J.P. Kennedy. Macromol. Symp. 2001, 175, 127. [e] C. Lin, I. Gitsov Macromolecules 2010, 43, 10017. [f] J. Tobis, L. Boch, Y. Thomann, J.C. Tiller J. Membr. Sci. 2011, 372, 219. [g] J. Kang, G. Erdődi, J.P. Kennedy, H. Chou, L. Lu, S. Grundfest-Broniatowski Macromol. Biosci. 2010, 10, 369. [h] N. Bruns, J.C. Tiller Nano Lett. 2005, 5, 45. [i] G. Savin, N. Bruns, Y. Thomann, J.C. Tiller, Macromolecules, 2005, 38, 7536. [j] N. Bruns, W. Bannwarth, J.C. Tiller Biotechnol. Bioeng. 2008, 101, 19. [k] S. Dech, T. Cramer, R. Ladisch, N. Bruns, J.C. Tiller Biomacromolecules 2011, 12, 1594. [I] S. Dech, V. Wruk, C.P. Fik, J.C. Tiller Polymer 2012, 53, 701. [m] S.K. Jewrajka, G. Erdődi, J.P. Kennedy, D. Ely, G. Dunphy, S. Boehme, F. Popescu J. Biomed. Mater. Res., Part A 2008, 87A, 69. [n] S. Rimmer, S.P. Wilshaw, P. Pickavance, E. Ingham Biomaterials 2009, 30, 2468. [o] A. Haesslein, 
M.C. Hacker, H. Ueda, D.M. Ammon, R.N. Borazjani, J.F. Küzler, J.C. Salamone, A.G. Mikos J. Biomater. Sci. Polym. Ed. 2009, 2, 49. [p] J.K. Xu, X.S. Li, F.Q. Sun Drug Delivery 2011, 18, 150. [q] M. Hanko, N. Bruns, J.C. Tiller, J. Heinze Anal. Bioanal. Chem.,2006, 386, 1273. [r] N. Bruns, M. Hanko, S. Dech, R. Ladisch, J. Tobis, J.C. Tiller Macromol. Symp. 2010, 291, 293. [s] Y. Wang, J.A. Finlay, D.E. Betts, T.J. Merkel, J.C. Luft, M.E. Callow, J.A. Callow, J.M. DeSimone Langmuir, 2011, 27, 10365. [t] Y. Wang, D.E. Betts, J.A. Finlay, L. Brewer, M.E. Callow, J.A. Callow, D.E. Wendt, J.M. DeSimone Macromolecules 2011, 44, 878. [u] J.W. Bartels, P.M. Imbesi, J.A. Finlay, C.F. Jun Ma, J.E. Seppala, A.M. Nystrom, M.E. Mackay, J.A. Callow, M.E. Callow, K.L. Wooley ACS Appl. Mater. Interfaces, 2011, 3, 2118.

19 [a] W. Lequieu, F.E. Du Prez Polymer, 2004,45, 749-757. [b] I. Aranaz, S. Carrasco, M.G. Tardajos, C. Elvira, H. Reinecke, D. Lopez, A. Gallardo Polym. Chem., 2011, 2, 709-713.

20 (a) Hoffman, A. S Adv. Drug Delivery Rev. 2013, 65, 10-16. (b) Sato, T; Tanka, K; Toyokura, A.; Mori, R.; Takashaki, R.; Terao, K.; Yusa, S. Macromolecules 2013, 46, 226-235. (c) Ding, A.; Lu, G.; Guo, H.; Zheng, X.; Huang, X. J. Polym. Sci., Part A: Polym. Chem. 2013, 51, 1091-1098. (d) Takahasi, A.; Hamai, K.; Okada, Y.; Sokohara, S. Polymer 2011, 52, 3791-3799. (e) Lee, J. S.; Feijen, J. J. Controlled Rel. 2012, 161, 473-483. (f) Li, Y. M.; Qian, Y. F.; Liu, T.; Zhang, G. Y., Liu, S. Y. Biomacromolecules 2012, 13, 3877-3886. (g) Hu, J. M. ; Dai, L.; Lin, S. Y. Macromolecules 2011, 44, 46994710. (h) Wei, H.; Cheng, S. X.; Zhang, X. S.; Zhuo, R. X. Prog. Polym. Sci. 2009, 34, 893-910. (i) Xu, F., Yan, T. T.; Luo, Y. L. J. Bioact. Comp. Polym. 2013, 28, 66-85. 
${ }^{21}$ L. Toman, M. Janata, J. Spevacek, J. Brus, A. Sikora, P. Latalova, P. Holler, P. Vlcek, B. Dvorankova J. Polym. Sci., Part A: Polym. Chem. 2006, 44, 6378.

22 J. P. Kennedy, B. Iván Designed Polymers by Carbocationic Macromolecular Engineering: Theory and Practice; Hanser Publishers: New York, 1992.

${ }^{23}$ B. Iván, J. P. Kennedy J. Polym. Sci., Part A: Polym. Chem. 1990, 28, 89.

${ }^{24}$ B. Iván, J. P. Kennedy, V. S. C. Chang. J. Polym. Sci., Polym. Chem. Ed. 1980, 18, 3177.

${ }^{25}$ Virtanen, J.; Tenhu, H. J. Polym. Sci., Part A: Polym. Chem. 2001, 39, 3716-3725.

${ }^{26}$ Lee, C. B.; Cho, C. G. Polymer-Korea 2000, 24, 168-173.

${ }^{27}$ W. Brostow, R. Chiu, I.M. Kalogeras, A. Vassilikou-Dova Materials Letters, 2008, 62, 3152.

${ }^{28}$ V. Mano, E. S. Ribeiro, E. S. Maria, N. Barbani, P. Giusti J App. Polym. Sci., 2004, 91, 501.

${ }^{29}$ M. Shibayama, T. Tanaka Advances in Polymer Science, 1993, 109, 1 (b) H. Cheng,

L. Shen, C. Wu Macromolecules 2006, 39, 2325.

${ }^{30}$ H.G. Schild, D.A. Tirrell Macromolecules, 1990, 94, 4352.

${ }^{31}$ H. Feil, Y. H. Baet J. Feijen, S.W. Kim Macromolecules 1993, 26, 2496.

${ }^{32}$ K. László, K. Kosik, E. Geissler Macromolecules, 2004, 37, 10067. 